\title{
Ueber \\ den reizbaren und leitenden Bestandtheil, sowie über die angebliche Unermüdbarkeit der Nervenfaser.
}

Von

\section{Eduard Pfïger.}

Die ungehevere Hochflut der medicinischen und naturwissenschaftlichen Veröffentlichungen der gegenwärtigen Zeit macht es einigermaassen begreiflich, aber nicht immer entschuldbar, dass die in Betracht kommende, in zugänglichen Zeitschriften enthaltene Literatur oft genug in unglaublicher Weise sogar von solchen Forschern vernachlässigt wird, welche auf dem betreffenden Gebiete als Specialforscher sich bethätigen. Und die Vernachlässigung trifft nicht bloss untergeordnete, sondern auch die wichtigsten Arbeiten der bekanntesten Forscher.

Das hat dann die Folge, dass gewisse Autoren Fragen behandeln und ihre Beantwortung veröffentlichen, ohne zu wissen, dass diese Fragen längst von mir, zuweilen sogar viel strenger, entschieden wurden.

Zur Wahrung meines geistigen Eigenthums will ich deshalb zuerst darthun, wie ich vor nunmehr 19 Jahren den Beweis geliefert habe, welcher Bestandtheil der Nervenfaser die reizbare und leitende Substanz darstellt. Ich sagte ${ }^{1}$ ):

„Es gibt, wie ich sehe, nur wenige Anhaltspunkte, um zu ent"scheiden, ob die Faser oder die Flüssigkeit in der Zelle das Princip "des Lebens enthalten.

„Betrachten wir zunächst den Nerven, der Axencylinder genannt "wird, so zeichnet er sich durch diejenige Eigenschaft, welche die "lebendige Materie zu allererst kennzeichnet, in hervorragender

1) Die allgemeinen Lebenserscheinungen S. 31. Emil Strauss, Bonn 1889. 
"Weise aus, das heisst durch ausserordentliche Reizbarkeit. Nun "ergibt die mikroskopische Untersuchung, wie zuerst Max Schultze "entdeckt hat, dass der Nerv noch aus einem Bündel feinster „Fäserchen zusammengesetzt ist, denen als den Elementen des Nerven "die Reizbarkeit zugesprochen werden muss. Das ist auch die „Ansicht aller Physiologen.

"Wenn man nun den Nerven nach dem Rückenmark weiter "verfolgt, so findet man, wie der frühere Bonner talentvolle am "8. December 1863 verstorbene Privatdozent Otto Deiters nach"wies, dass der Axencylinder in einer Nervenzelle des Rückenmarks „oder Gehirns endigt, und dass, wie Max Schultze darthat, die „Fibrillen der Nervenfaser in die Fibrillen des Protoplasmas der "Nervenzelle übergehen. Folglich sind die Fäserchen des Proto"plasmas reizbare Substanz.

„Da zwischen den Fibrillen der Nervenfasern gewiss noch "flüssiger Saft sich findet, wie zwischen den Fibrillen der Nerven"zelle, so könnte man das Bedenken haben, dass vielleicht trotz "Allem die Flüssigkeit das Reizbare sei und nicht das Fäserchen."

Ich will hier einfügen, dass hervorragende Anatomen, wie F. Leydig, in dem Saft der Nervenfaser die reizbare Substanz erblicken wollen.

Ich fuhr dann weiter fort.

„Es hat nun bereits im vorigen Jahrhundert (1797) Galvani ${ }^{1}$ ), „der Entdecker der elektrischen Ströme in dem lebendigen Orga„nismus, eine Thatsache gefunden, die von grosser Bedeutung „für die vorliegende Frage ist.

„Die Nerven werden bekanntlich durch die allerschwächsten „elektrischen Ströme gereizt. Galvani zeigte nun, dass ein „elektrischer Strom einen Nerven nicht erregt, wenn er ihn quer „durchfliesst. Liegt die Nervenfaser genau senkrecht auf der „Richtung des Stromes, so kömnen sogar starke Induktions,schläge durch ihn geschickt werden, ohne dass Erregung eintritt „Diese Abhängigkeit der Reizung von dem Winkel, den die „Richtung des elektrischen Stromes mit den Nervenfibrillen macht, „ist ein starker Grund für die Annahme, dass die Fibrille und "nicht der Saft die reizbare Substanz ist. Die Fibrillen der

1) Galvani, Opere edite et inedite etc. Seconda memoria allo Spallanzani 1797 s. $341,347$. 
„Nerven sind aber die stetigen Fortsetzungen der Fibrillen der "Nervenzelle.

„Dass die Fibrillen der Nervenzelle und nicht der Saft der„selben in Uebereinstimmung mit dem vorigen Resultat das physi„sche Substrat sogar der psychischen Processe darstellen, lässt ,sich auch noch anf anderem Wege vertheidigen.

„Das Erinnernngsbild in unserem Gelirn ist die bewusst „werdende Spur, welche die Sinneserregung in den Nervenzellen „des Gehirns zurückgelassen hat. Da gewisse Erkrankungen der „Gehirnmaterie den Gedächtnissinhalt bald in kleinerem, bald in ,grösserem Umfange verlösehen, muss das Erinnerungsbild an „ein materielles Substrat gebunden sein. Man kann sich nun die „danernde Fixation eines Bildes wohl in fester, nicht in flüssiger „Materie denken. Ich muss deshalb das physische Substrat der „Erinnerung in die organisirten Theile der Gehirnzellen, d. h. ,in die Fibrillen verlegen. - -

„Eine weitere Bestätignng unserer Auffassung anf anderem „Gebiete liegt darin, dass das für den Nerven vorher angeführte „Galvani'sche Gesetz sich anch für die Muskelzellen bewährt „hat, wie Prof. L. Herma nn und Dr. Gi i for é ${ }^{1}$ ) nachwiesen. „Die Muskelfaser ist eine dicke, lange, cylindrische, mehrkernige „Zelle, deren Protoplasma theilweise wie beim Nerven aus parallel „neben einander verlaufenden Fibrillen besteht, die von Flüssig„keit durchtränkt and umspült sind. Hiernach ist also wie beim „Nerven nicht der Saft des Muskels, sondern die Faser, d. h. die „feste organisirte Materie das Reizbare."

In jüngster Zeit ist von Albrecht Bethe ${ }^{2}$ ) noch eine sinnreiche Untersuchung veröffentlicht worden, welche die leitende Funetion der Neurofibrillen in überzeugender Art beweist. Dieser Forseher verwerthete den in der Nervenfaser wellenförmigen Verlauf der Neurofibrillen, indem er die Fortpflanzungsgeschwindigkeit der Reizung bei verschiedener Spannung einer bestimmten Nervenlänge

1) Jacob Albrecht, August Meyer und Liborio Giuffré, Untersuchungen über die Erregbarkeit der Nerven und Muskeln bei Längs- und Querdurchströmung. Dies Archiv Bd. 21 S. 467. 1879.

2) Albrecht Bethe, Ein neuer Beweis für die leitende Function der Neurofibrillen, nebst Bemerkungen über die Reflexzeit, Hemmungszeit und Latenzzeit des Muskels beim Blutegel. Dies Archiv Bd. 122 S. 35.1908. 
maass. Diese Nervenlänge nimmt mit der Spannung zu, während die Länge der Fibrillen natürlich unverändert bleibt.

Als Ergebniss hebt Albrecht Bethe hervor:

"In dehnbaren Nerven (Hirudo) ist die Leitungsngeschwindigkeit in einem gegebenen Nervenstück "proportional seiner jeweiligen Länge, solange die "Dehnung innerhalb der physiologischen Grenzen „bleibt. Da die Neurofibrillen innerhalb der physio"logischen Dehnungsgrenzen die gleiche Länge be„wahren, so sind dieselben als die Hauptträger der "Erregungsleitung anzusehen." Die Leitungsgeschwindigkeit in den Fibrillen ergab sich für den Blutegel $\mathrm{zu} 32-40 \mathrm{~cm}$ in der Secunde.

Meines Erachtens sind die von mir vor zwei Decennien angeführten Gründe auch heute noch gültig. Sie bestätigen, was ja, aus anderen Gesichtspunkten betrachtet, nicht zweifelhaft sein kann, dass der Lebensprocess gebunden ist an die organisirte Materie, d. b. an die nach bestimmten Gesetzen mit einander verknüpften und geordneten Molekülgruppen.

Eine andere wichtige Frage, die ich ebenfalls seit langer Zeit in strengster Form beantwortet habe, wird vielfach aufs Neue und zum Theil in recht mangelhafter Weise behandelt.

Die Nervenfaser soll nach der Ansicht einiger Forscher unermüdbar sein!

Bereits 1891 sagte ich ${ }^{\mathbf{1}}$ ):

"Ich will nicht unterlassen, hervorzuheben, dass ich bei der „Erklärung der Ermüdung die lähmende Eigenschaft der durch die "Arbeit erzeugten Zersetzungsstoffe keineswegs in Abrede stellen "will. Ich glaube aber nicht, dass hierin die wesentliche Ursache nder Ermüdung liegt, wofür auch das starke Sinken der Oxydations"processe des lebendigen Körpers in den Zeiten der Ruhe spricht, "welche einer anstrengenden Arbeit nachfolgt.

"Was mich zu der Ansicht, dass das Eiweiss, bzw. eine be"stimmte Eiweissart, die reizbare Materie sei, von jeher ganz be"sonders bestimmt hat, ist, dass die Richtung des elektrischen

1) E. Pflüger, Einige Erklärungen, betreffend meinen Aufsatz: Die Quelle der Muskelkraft. Dies Arch. Bd. 50 S. 337. 1891. 
„Stromes gegen die Fasern der Nerven und Maskeln von so. "grosser Bedentung für die Reiznng ist. Die Reizbarkeit ist „also eine Eigenschaft der Faser, der geformten Materie, der „organisirten Moleküle ${ }^{1}$ ). Alles eigentliche Leben hängt doch "unzweifelhaft an den nach bestimmten Gesetzen geordneten Molekül"gruppen. Reizbarkeit ist aber nicht denkbar ohne einen Inhalt an „potentieller Energie, und die organisirte Faser sowie das Organisirte „überhaupt ist nach allen seinen Reactionen immer für Eiweiss an"gesehen worden. Substitutionen verschiedener Art sind selbst„verständlich nicht ausgeschlossen.

„Die Ansicht einiger nenerer Forscher von der Unermïdlich"keit der Nerven, die mit allen übrigen gaten Thatsachen der "Physiologie in Widerspruch steht, wird schon durch die Ueber"legung, dass die Reizung der Nerven mit Entladung elektrischer "Ströme verknüpft ist, widerlegt. Denn jeder elektrische Strom „erzengt Wärme und bedingt einen Stoffverbranch. Ebenso ist "die tiefe Uebereinstimmung, die in den elektrophysiologischen "Gesetzen zwischen Maskel und Nerv besteht, ganz unvereinbar „mit der Ansicht, dass in der wichtigsten Eigenschaft beide "Gewebe sich so grundsätzlich unterscheiden sollten."

Meine soeben nochmals vorgetragene Beweisführung für die Ermüdbarkeit der Nerven ist vollkommen unangreifbar. Denn der Einwand, dass zwischen Actionsstrom und der Erregung keine nothwendige Beziehung bestehe, ist obne jede Berechtigung. -

Es ist aber vielleicht nicht unzweckmässig, wenn ich darzulegen versuche, weshalb immer aufs Neue bald hier, bald dort die Ansicht der Unermüdbarkeit der Nerven wieder Vertreter findet.

Obwohl der Muskel während seiner Thätigkeit einen sehr starken Stoffverbrauch hat, was ja Niemand bezweifelt, liefert er dennoch Beispiele, die den Findruck machen, als ob auch dieses Organ unermüdbar sei. Scheinbar glaubwürdige Berichte bezeugen, dass ein gezeichneter Albatros 8 Tage und 8 Nächte in der Luft einem Dampfer auf dem Meere gefolgt ist. - Das Herz des Menschen schlägt bei manchen auserwählten Individuen 100 Jahre lang. Bei

1) Der Leser sieht, dass ich den Beweis für die leitende Funktion der Fibrillen zwei Mal veröffentlicht habe, und zwar 1889 und 1891 also sogar in diesem Archive, so dass wenigstens den Physiologen volle Gelegenheit gegeben war, diesen Beweis kennen zu lernen.

E. Pflüger, Archiv für Physiologie. Bd. 122. 
598 Eduard Pflüger: Ueber den reizbaren und Ieitenden Bestandtheil etc.

dem Hund beträgt die Zahl der Pulse in der Minute 90-100. Schläge, bei dem Kaninchen 140-240 und soll bei dem Eichhörnchen sogar bis 500 steigen können. Damit ist bewiesen, dass der kurze Augenblick zwischen zwei Herzschlägen genügt, um trotz des bedeutenden Kraftverbrauches vollkommene Erholung zu ermöglichen. Diese Erholung vollzieht sich unzweifelhaft auf Kosten der Nährstoffe der Gewebssäfte, die die Muskelfibrillen umspülen und durchtränken. Bei den Nerven liegen die Verhältnisse für die Erzeugung eines Anscheines 'von Unermüdbarkeit noch viel günstiger. Denn die Grösse des Kraftaufwandes, welche sich mit der Nervenerregung verknüpft, ist ja so klein, dass es bis jetzt nicht gelungen ist, eine durch sie bedingte Temperatursteigerung nachzuweisen. Hier reicht also der die Neurofibrillen umspülende ernährende Saft noch sehr viel länger aus.

Es ist bemerkenswerth, dass der Schein der Unermüdbarkeit auch bei Erregungen sich geltend macht, die offenbar an die Substanz der centralen Ganglienzelle gebunden siud. Ich meine den Schmerz, bei dem ebenfalls ein rascher Ersatz der verbrauchten Nervensubstanz gewährleistet ist. Der tiefere Sinn dieser Einrichtung liegt darin, dass der Schmerz das Individuum zwingt, zur Beseitigung der Schädigung das Äusserste zu versuchen. Wenn es sich um die Sicherung der Existenz des Individuums oder der Art handelt, stossen wir immer auf dahin gerichtete Einrichtungen. - Es kommen aber auch Fälle vor, wo die Ermüdbarkeit des Nervensystemes ziemlich leicht bemerkbar wird, wie z. B. bei der Betrachtung einer homogenen Farbe. Man muss hier daran denken, dass bei dem gewöhnlichen Gebrauch des Sehorganes im Wesentlichen nur Mischfarben in Betracht kommen, so dass die Einwirkung der homogenen Farbe nur einen Ausnahmezustand bildet. Es erwächst deshalb aus dieser schwachen Seite des Sehorganes dem Individuum keinerlei Nachtheil. 